\title{
Ratios of heavy baryons to heavy mesons in relativistic nucleus-nucleus collisions
}

\author{
Yongseok Oh,,${ }^{1 *}$ Che Ming Ko, ${ }^{1, \dagger}$ Su Houng Lee, ${ }^{2, \ddagger}$ and Shigehiro Yasui ${ }^{3, \S}$ \\ ${ }^{1}$ Cyclotron Institute and Physics Department, Texas A\&M University, College Station, Texas 77843, USA \\ ${ }^{2}$ Institute of Physics and Applied Physics, Yonsei University, Seoul 120-749, Korea \\ ${ }^{3}$ High Energy Accelerator Research Organization (KEK), Tsukuba, Ibaraki 302-0801, Japan
}

(Received 14 January 2009; published 15 April 2009)

\begin{abstract}
Heavy baryon/meson ratios $\Lambda_{c} / D^{0}$ and $\Lambda_{b} / \bar{B}^{0}$ in relativistic heavy ion collisions are studied in the quark coalescence model. For heavy baryons, we include production from coalescence of heavy quarks with free light quarks as well as with bounded light diquarks that might exist in the strongly coupled quark-gluon plasma produced in these collisions. Including the contribution from decays of heavy hadron resonances and also that due to fragmentation of heavy quarks that are left in the system after coalescence, the resulting $\Lambda_{c} / D^{0}$ and $\Lambda_{b} / \bar{B}^{0}$ ratios in midrapidity $(|y| \leqslant 0.5)$ from central $\mathrm{Au}+\mathrm{Au}$ collisions at $\sqrt{s_{N N}}=200 \mathrm{GeV}$ are about a factor of five and ten, respectively, larger than those given by the thermal model, and about a factor of ten and twelve, respectively, larger than corresponding ratios in the PYTHIA model for $p p$ collisions. These ratios are reduced by a factor of about 1.6 if there are no diquarks in the quark-gluon plasma. The transverse momentum dependence of the heavy baryon/meson ratios is found to be sensitive to the heavy quark mass, with the $\Lambda_{b} / \bar{B}^{0}$ ratio being much flatter than the $\Lambda_{c} / D^{0}$ ratio. The latter peaks at the transverse momentum $p_{T} \simeq 0.8 \mathrm{GeV}$ but the peak shifts to $p_{T} \simeq 2 \mathrm{GeV}$ in the absence of diquarks.
\end{abstract}

DOI: 10.1103/PhysRevC.79.044905

PACS number(s): 25.75.Cj, 25.75.Dw

\section{INTRODUCTION}

Recent measurements on nonphotonic electrons from decays of midrapidity heavy-flavored mesons in central heavy ion collisions at the Relativistic Heavy Ion Collider (RHIC) have shown that the nuclear modification factor $R_{A A}^{e}$ relative to $p p$ collisions is significantly smaller than theoretical predictions based on radiative energy loss of heavy quarks in produced quark-gluon plasma [1-4]. To explain the observed small value of $R_{A A}^{e}$ or large suppression of heavy meson production in relativistic heavy ion collisions, other mechanisms for energy loss of heavy quarks in quark-gluon plasma have been proposed [5-10]. Also, it was suggested that the enhancement of charmed baryon production over charmed meson production, i.e., the $\Lambda_{c} / D^{0}$ ratio, would suppress the $R_{A A}^{e}$ of the electrons from heavy mesons $[11,12]$. This is due to the fact that enhanced charmed baryon production reduces the production of charmed mesons and thus their contribution to decay electrons.

In fact, experiments at RHIC have shown that in central heavy ion collisions there is an enhanced production of midrapidity baryons in the intermediate transverse momentum region not only in light hadrons but also in strange hadrons. This enhancement could be described by multiquark dynamics through quark coalescence or recombination [13-15], baryon junction loops [16-18], or long-range coherent fields [19]. Therefore, one might expect enhanced heavy baryon production in the intermediate transverse momentum region as well.

\footnotetext{
*yoh@comp.tamu.edu

†ko@comp.tamu.edu

${ }^{\ddagger}$ suhoung@phya.yonsei.ac.kr

§yasuis@post.kek.jp
}

Since the $\Lambda_{c} / D^{0}$ ratio has not been measured in heavy ion collisions, it was assumed in Ref. [11] to be the same as the measured $\Lambda / K_{S}^{0}$ ratio or in Ref. [12] to have a Gaussian form with a maximum value peaked at the transverse momentum $p_{T}=5 \mathrm{GeV}$. It was then claimed that a value of $\Lambda_{c} / D^{0} \sim 1$, which is about a factor of ten larger than that in $p p$ collisions at same energy, could result in an additional 20-25\% suppression of $R_{A A}^{e}$ apart from the suppression due to the collisional energy loss of heavy quarks in quark-gluon plasma. However, it is not yet known what kind of underlying physics can cause such a large enhancement of the $\Lambda_{c} / D^{0}$ ratio.

Recently, enhancement of the $\Lambda_{c} / D^{0}$ ratio due to the existence of $[u d]$ diquarks in strongly coupled quark-gluon plasma (QGP) was suggested by Lee et al. [20]. This was based on the idea of possible existence of quasi bound states of quarks and gluons including diquarks in QGP when the temperature is between $T_{C}$ and $4 T_{C}$ [21], where $T_{C}$ is the critical temperature for the quark-gluon plasma to hadronic matter transition. Based on the quark coalescence model, it was shown in Ref. [20] that the $\Lambda_{c} / D^{0}$ ratio could be enhanced by a factor of 4-8 relative to the case without diquarks in QGP, depending on the binding energy of the light diquark at $T_{C}=175 \mathrm{MeV}$. The question whether diquarks can exist as quasi bound states in cold quark matter was raised earlier in Ref. [22], where the quark-diquark matter was claimed to be energetically more favorable than the free quark matter. The idea of enhancement of $\Lambda_{c}$ baryon production in heavy-ion collisions was then suggested in Ref. [23]. Based on the assumption that the number of diquarks present in a collision is mainly determined by that in the initial nuclei, the author of Ref. [23] considered the $\Lambda_{c} / \Sigma_{c}$ ratio and estimated that it could be enhanced by a factor of as large as 80 . Since the $\Sigma_{c}$ cannot be directly measured in near-future heavy-ion experiments, the more phenomenologically accessible $\Lambda_{c} / D^{0}$ ratio is thus addressed in Ref. [20]. 
The idea of diquarks [24-26] has been widely used in hadron physics for describing the mass spectrum, electromagnetic properties, and many other properties of hadrons (for a review, see, for example, Ref. [27]), although the properties and even the existence of diquarks are still under debate [28]. Among possible signatures of diquarks in hadrons, the observed large ratio of $\Lambda_{c} / \Sigma_{c} \sim 7$ in $e^{+} e^{-}$collisions was pointed out in Ref. [29], which implies a significantly larger production of scalar diquarks than vector diquarks [30]. (See also Ref. [31].) In spite of the lack of concrete consensus on the diquark nature, it is well-known that the (isoscalar) scalar light $[u d]$ diquark of color antitriplet is the most probable diquark state. Evidence for such scalar diquarks has been confirmed by a recent lattice QCD simulation [32]. (See, however, Ref. [33] for other lattice QCD calculation on diquarks.)

In diquark models, the $\Lambda_{c}$ is usually considered as a system consisting of a charm quark and a scalar light $[u d]$ diquark. On the other hand, the structure of $\Sigma_{c}$ and $\Sigma_{c}^{*}$ is model-dependent: they may have an axial-vector light diquark but they also may contain diquarks $[Q q]$ made of one heavy quark $(Q)$ and one light quark $(q)[34,35]$. In Ref. [36], it is claimed that the ground state baryons favor the $[Q q]$ diquark. This is based on the observation that the $[Q q] q$ system gives a $\Lambda_{c}$ mass closer to that from the three-quark calculation, although it has a larger mass than that based on the $Q[q q]$ configuration. Since the color-spin interaction between quarks is inversely proportional to the quark masses, the binding energies of $[Q q]$ diquarks are smaller than those of scalar light $[q q]$ diquarks, and it is not clear whether such $[Q q]$ diquarks can exist, in particular, in QGP. (See Ref. [37] for a recent discussion on the diquark picture for heavy baryons.)

In this paper, we calculate the $\Lambda_{Q} / H^{0}$ ratios $(Q=b, c$ and $H^{0}$ stands for $D^{0}$ or $\bar{B}^{0}$ ) in relativistic heavy ion collisions based on the quark coalescence model. We consider the two cases of $\Lambda_{Q}$ being a pure three-quark state and of $\Lambda_{Q}$ being made of a heavy quark and one scalar light diquark. In addition to the direct formation of $\Lambda_{Q}$ and $H^{0}$, we also take into account the contribution from decays of heavy hadron resonances. Namely, we consider the decay of $D^{*}$ mesons for $D^{0}$ production and $\Sigma_{Q}$ and $\Sigma_{Q}^{*}$ baryon decays for $\Lambda_{Q}$ production. As we have discussed before, we assume that $\Sigma_{Q}$ and $\Sigma_{O}^{*}$ baryons are made of three quarks and that neither vector light diquarks nor scalar heavy-light diquark exists in QGP.

This paper is organized as follows. In the next section, we briefly discuss heavy hadron production in $p p$ collisions. A simple thermal model is then used in Sec. III to show the role of heavy hadron resonances in the $\Lambda_{Q} / H^{0}$ ratios. The difference between the ratios for charmed hadrons and for bottom hadrons is also discussed. In Sec. IV, the coalescence model used in this work is given together with both light and heavy quark distribution functions. Also described are the calculational methods and the contributions from fragmentation of heavy quarks that are not used in coalescence. We then show in the same section the results for the relative production fractions of heavy hadrons and their $p_{T}$ spectra as well as the transverse momentum dependence of the $\Lambda_{Q} / H^{0}$ ratios. Section $V$ contains the conclusions and discussions.

\section{HEAVY HADRON PRODUCTION IN PROTON-PROTON COLLISIONS}

Before studying the production of heavy hadrons in heavy ion collisions, we briefly discuss their production in $p p$ collisions by using the PYTHIA model [38]. For $p p$ collisions at $\sqrt{s}=200 \mathrm{GeV}$, the PYTHIA model gives the following relative production fractions of charmed hadrons in midrapidity $(|y| \leqslant 0.5)^{1}$ :

$$
\begin{array}{ll}
f\left(D^{+}\right) \simeq 0.196, \quad & f\left(D^{0}\right) \simeq 0.607, \\
f\left(D_{s}^{+}\right) \simeq 0.121, & f\left(\Lambda_{c}\right) \simeq 0.076 .
\end{array}
$$

This leads to the particle number ratios

$$
\left(\frac{D^{0}}{D^{+}}\right)_{p p} \simeq 3.1, \quad\left(\frac{\Lambda_{c}}{D^{0}}\right)_{p p} \simeq 0.13
$$

The above estimated $D^{0} / D^{+}$ratio can be understood largely by considering resonance decays, namely, decays of $D^{*}$ mesons into $D$ mesons. First, the $D^{* 0}$ meson decays into $D^{0}$ meson by $100 \%$ [39] since the decay of $D^{* 0}$ into $D^{+} \pi^{-}$is prohibited by energy conservation. ${ }^{2}$ Second, the $D^{*+}$ meson can decay either into $D^{+}$or $D^{0}$. The branching ratios are $B R\left(D^{*+} \rightarrow D^{0} \pi^{+}\right) \approx 68 \%$ and $B R\left(D^{*+} \rightarrow D^{+} X\right) \approx 32 \%$, where sum over any state $X$ is understood. If the production rates are equal for $D$ and $D^{*}$ mesons aside from the factor due to different spin degeneracies, we then would have

$$
\left(\frac{D^{0}}{D^{+}}\right)_{p p} \approx \frac{1+3+3 \times 0.68}{1+3 \times 0.32}=\frac{6.04}{1.96} \approx 3.1
$$

where the spin degeneracy of $D^{*}$ is included. We also note that the estimated $\Lambda_{c} / D^{0}$ ratio is close to that $(\approx 0.16)$ in the SELEX measurement [40].

For bottom hadron production in $p p$ collisions at $\sqrt{s_{N N}}=$ $200 \mathrm{GeV}$, the relative production fractions in midrapidity from the PYTHIA model are

$$
\begin{aligned}
f\left(B^{-}\right) & \simeq 0.101, \quad f\left(\bar{B}^{0}\right) \simeq 0.101, \quad f\left(B_{s}^{-}\right) \simeq 0.030, \\
f\left(B^{*-}\right) & \simeq 0.302, \quad f\left(\bar{B}^{* 0}\right) \simeq 0.301, \quad f\left(B_{s}^{*-}\right) \simeq 0.089, \\
f\left(\Lambda_{b}\right) & \simeq 0.076,
\end{aligned}
$$

leading to $^{3}$

$$
\Lambda_{b} / \bar{B}^{0} \approx 0.7
$$

\footnotetext{
${ }^{1}$ We have confirmed the results reported in Ref. [12]. These values are also close to the values quoted by Particle Data Group (PDG) [39] for $e^{+} e^{-}$annihilation at $\sqrt{s}=91 \mathrm{GeV}: f\left(D^{+}\right) \simeq 0.21, f\left(D^{0}\right) \simeq$ $0.54, f\left(D_{s}^{+}\right) \simeq 0.16$, and $f\left(\Lambda_{c}\right) \simeq 0.093$.

${ }^{2}$ Note that $m\left(D^{* 0}\right)=2007 \mathrm{MeV}, m\left(D^{*+}\right)=2010 \mathrm{MeV}, m\left(D^{0}\right)=$ $1865 \mathrm{MeV}, m\left(D^{+}\right)=1869 \mathrm{MeV}$, while $m\left(\pi^{ \pm}\right)=139.6 \mathrm{MeV}$ and $m\left(\pi^{0}\right)=135 \mathrm{MeV}$.

${ }^{3}$ We note that the average fractions of bottom mesons and bottom baryons in $p \bar{p}$ annihilation at $\sqrt{s}=1.5 \mathrm{TeV}$ have been estimated to be $f(b \bar{u})=f(b \bar{d})=0.399$ and $f$ (baryon) $=0.092$ [39]. If we assume that $b \bar{q}$ mesons include equal number of $B$ and $B^{*}$ mesons apart from the spin degeneracy and the number of bottom baryons is mostly due to $\Lambda_{b}$, then we would have $\Lambda_{b} / \bar{B}^{0} \approx 1.1$ in $p \bar{p}$ collisions.
} 
which is significantly larger than the $\Lambda_{c} / D^{0}$ ratio given in Eq. (2).

\section{THE THERMAL MODEL}

In relativistic heavy-ion collisions, the $\Lambda_{c} / D^{0}$ and $\Lambda_{b} / \bar{B}^{0}$ ratios are expected to be different from those given in Eqs. (2) and (5) for $p p$ collisions. This can be seen using a simple thermal model which assumes that in relativistic heavy ion collisions charmed and bottom hadrons are produced during hadronization of the quark-gluon plasma and that they are in both thermal and chemical equilibrium at the phase transition temperature $T_{C}$. The total charm and bottom numbers are, however, determined by initial hard scattering as thermal production of heavy quarks from created quark-gluon plasma is negligible in heavy ion collisions at RHIC [41]. The number of heavy hadrons of mass $m$ inside a fireball of volume $V$ at $T_{C}$ is then given by

$$
N=\gamma_{Q} \frac{g V}{2 \pi^{2}} m^{2} T K_{2}\left(m / T_{C}\right),
$$

where $\gamma_{Q}$ is the heavy-quark fugacity, $g$ is the degeneracy of the particle, and $K_{2}$ is the modified Bessel function.

For charmed hadrons, we would then have $\left(D^{0} / D^{+}\right)_{0}=1$, where the subscript 0 means the ratio without resonance contributions, if the $D^{*} \rightarrow D$ decay is not included. Since

$$
\frac{D^{* 0}}{D^{0}}=\frac{3 m_{D^{*}}^{2} K_{2}\left(m_{D^{*}} / T_{C}\right)}{m_{D_{0}}^{2} K_{2}\left(m_{D_{0}} / T_{C}\right)} \simeq 1.47
$$

at $T_{C}=175 \mathrm{MeV}$, including decays of $D^{*}$ mesons changes the ratio to

$$
\frac{D^{0}}{D^{+}} \approx \frac{1+(1+0.68) \times 1.47}{1+0.32 \times 1.47} \approx 2.36,
$$

if we assume that the same branching ratios for $D^{*}$ decay to $D$ as in free space. The $D^{0} / D^{+}$ratio in heavy-ion collisions is thus about $25 \%$ smaller than that in $p p$ collisions.

For the $\Lambda_{c} / D^{0}$ ratio, we have, by using $m_{\Lambda_{c}}=2285 \mathrm{MeV}$ and $m_{D^{0}}=1865 \mathrm{MeV}$,

$$
\left(\frac{\Lambda_{c}}{D^{0}}\right)_{0}=\frac{2 m_{\Lambda_{c}}^{2} K_{2}\left(m_{\Lambda_{c}} / T_{C}\right)}{m_{D_{0}}^{2} K_{2}\left(m_{D_{0}} / T_{C}\right)} \simeq 0.24
$$

where the factor 2 is the spin degeneracy of $\Lambda_{c}$ baryon. Since the ratio $D^{*} / D$ is 1.47 , inclusion of $D^{*}$ resonance decays causes a strong reduction of the $\Lambda_{c} / D^{0}$ ratio. On the other hand, baryon resonances can also contribute to the production of $\Lambda_{c}$ baryon through their decays and can enhance the $\Lambda_{c} / D^{0}$ ratio. The major resonance contribution to $\Lambda_{c}$ comes from $\Sigma_{c}^{*}(2520)$ of spin-3/2. In thermal model, we have

$$
\frac{\Sigma_{c}^{*}(2520)}{\Lambda_{c}} \approx 2 \times 3 \times \frac{m_{\Sigma_{c}^{*}}^{2} K_{2}\left(m_{\Sigma_{c}^{*}} / T_{C}\right)}{m_{\Lambda_{c}}^{2} K_{2}\left(m_{\Lambda_{c}} / T_{C}\right)}=1.8,
$$

where the factor 2 comes from the difference in the spin degeneracies of $\Lambda_{c}$ and $\Sigma_{c}^{*}$, and the factor 3 is due to the isospin 1 of $\Sigma_{c}^{*}$. Since $\Sigma_{c}^{*}(2520)$ decays almost $100 \%$ into $\Lambda_{c}$, inclusion of its contribution gives $180 \%$ enhancement to the number of $\Lambda_{c}$. Another important contribution to $\Lambda_{c}$ production is from the decay of $\Sigma_{c}$ ground state, $\Sigma_{c}(2455)$ of spin-1/2, via the $\Sigma_{c} \rightarrow \Lambda_{c} \pi$ decay. ${ }^{4}$ In thermal model, its abundance relative to that of $\Lambda_{c}$ is

$$
\frac{\Sigma_{c}(2455)}{\Lambda_{c}} \approx 3 \times \frac{m_{\Sigma_{c}}^{2} K_{2}\left(m_{\Sigma_{c}} / T_{C}\right)}{m_{\Lambda_{c}}^{2} K_{2}\left(m_{\Lambda_{c}} / T_{C}\right)}=1.26 .
$$

Therefore, the contribution from $\Sigma_{c}$ (2455) and $\Sigma_{c}^{*}(2520)$ decays increases the $\Lambda_{c}$ number by a factor of 3.1 and brings the $\Lambda_{c}$ to $D^{0}$ ratio close to the naive expectation given in Eq. (9) based only on directly produced $\Lambda_{c}$ and $D^{0}$, i.e.,

$$
\begin{aligned}
\frac{\Lambda_{c}}{D^{0}} & =\left.\frac{\Lambda_{c}\left\{1+\Sigma_{c}^{*}(2520) / \Lambda_{c}+\Sigma_{c}(2455) / \Lambda_{c}\right\}}{D^{0}\left(1+1.68 D^{*} / D\right)}\right|_{\text {ther }} \\
& \simeq\left(\frac{\Lambda_{c}}{D^{0}}\right)_{0} \frac{1+1.8+1.26}{1+1.68 \times 1.47} \approx 0.28 .
\end{aligned}
$$

The contribution from higher mass charmed resonances is negligibly small in the thermal model. For $D$ meson production, the next higher state is $D_{1}(2420)$ of $J^{P}=1^{+}$. The branching ratio of the decay of this meson is not well-known except that it decays into $D^{*} \pi$ but not into $D \pi$. If we assume that it decays into $D^{0}$ via an intermediate $D^{*}$, i.e., $D_{1} \rightarrow D^{*} \pi \rightarrow D \pi \pi$, the denominator of Eq. (12) is then modified by the addition of $1.68 \times 3 \times 0.06 \simeq 0.3$, where the factor 0.06 is the $D_{1}(2420) / D^{0}$ ratio, giving thus less than $10 \%$ enhancement to $D^{0}$ production. For higher baryon resonances, the next excited state is $\Lambda_{c}$ (2593). Its contribution to the numerator of Eq. (12) is about 0.2 , since there is no difference in its spin and isospin degeneracy from that of $\Lambda_{c}$. Thus it again gives an effect less than $5 \%$ to $\Lambda_{c}$ production. The final value of the $\Lambda_{c} / D^{0}$ ratio then essentially does not change, i.e., the ratio is modified from 0.28 to 0.27 , which is about twice of that in $p p$ collisions.

The estimated value of the $\Lambda_{c} / D^{0}$ ratio from our simple thermal model is close to the value obtained in the more sophisticated thermal or statistical model of Ref. [42], which gives a charmed baryon to meson ratio $(c q q) /(c \bar{q}) \leqslant 0.25$. It is also consistent with the statistical model predictions of Ref. [43], which has $\Lambda_{c} / D^{0} \sim 0.2$ at the RHIC energy.

The $\Lambda_{b} / \bar{B}^{0}$ ratio in the thermal model can be estimated in the same way as for the $\Lambda_{c} / D^{0}$ ratio. The main difference between $\bar{B}^{0}$ and $D^{0}$ productions is that the bottom quark is much heavier than the charm quark. As a result, the mass difference between $B^{*}$ and $B$ is much smaller than that between $D^{*}$ and $D$ because of the manifestation of heavy quark spin symmetry. In fact, the mass difference between $D^{*}$ and $D$ is about $140 \mathrm{MeV}$, while that between $B^{*}$ and $B$ is only [39]

$$
m\left(B^{*}\right)-m(B) \approx 5325 \mathrm{MeV}-5279 \mathrm{MeV}=46 \mathrm{MeV} .
$$

\footnotetext{
${ }^{4}$ In the strange quark sector, the small mass difference between $\Lambda$ and $\Sigma$ does not allow the strong decay of $\Sigma$ into $\Lambda$, and only $\Sigma^{0} \rightarrow \Lambda \gamma$ is allowed. But in heavy quark sector, the mass difference between $\Lambda_{c}$ and $\Sigma_{c}$ is well above the pion mass.
} 
Therefore, unlike $D^{0}$ production, $B^{*}$ mesons cannot decay into $B$ mesons by strong interaction. ${ }^{5}$ This leads to a large suppression of $B$ meson production compared with the case for $D$ meson production. With $m\left(\Lambda_{b}\right)=5620 \mathrm{MeV}$, our simple thermal model then gives

$$
\frac{B^{*+}}{B^{+}} \approx 0.78, \quad\left(\frac{\Lambda_{b}}{\bar{B}^{0}}\right)_{0} \approx 0.32
$$

It indeed shows that $\left(\Lambda_{b} / \bar{B}^{0}\right)_{0}$ is somewhat larger than $\left(\Lambda_{c} / D^{0}\right)_{0}$.

Unlike $B$ production, the production of $\Lambda_{b}$ is affected by resonance decays. This is because the mass difference between $\Lambda_{b}$ and $\Sigma_{b}$ approaches to a finite value $(\sim 195 \mathrm{MeV}$ [44]) in the infinite mass limit, although the mass difference between $\Sigma_{b}$ of spin-1/2 and $\Sigma_{b}^{*}$ of spin-3/2 becomes smaller as the heavy quark mass increases. By using the recent experimental information for the $\Sigma_{b}$ baryons [45],

$$
M\left(\Sigma_{b}\right)=5812 \mathrm{MeV}, \quad M\left(\Sigma_{b}^{*}\right)=5833 \mathrm{MeV},
$$

the $\Lambda_{b} / \bar{B}^{0}$ ratio becomes

$$
\frac{\Lambda_{b}}{\bar{B}^{0}}=\left(\frac{\Lambda_{b}}{\bar{B}^{0}}\right)_{0}(1+3 \times 0.35+3 \times 2 \times 0.31) \approx 1.25,
$$

where the factors 0.35 and 0.31 are the $\Sigma_{b} / \Lambda_{b}$ and $\Sigma_{b}^{*} / \Lambda_{b}$ ratios, respectively. The $\Lambda_{b} / \bar{B}^{0}$ ratio is seen to be larger than the $\Lambda_{c} / D^{0}$ ratio by a factor of about 5 . Compared to its value in $p p$ collisions, the $\Lambda_{b} / \bar{B}^{0}$ ratio in thermal model for heavy ion collisions is close to a factor of two larger, similar to the case for the $\Lambda_{c} / D^{0}$ ratio.

There are other higher charmed and bottom resonances listed by the PDG [39]. These include heavy baryons that contain strange quarks such as $\Xi_{Q}, \Xi_{Q}^{\prime}, \Omega_{Q}$, and their resonances. Although most of corresponding bottom baryons are yet to be discovered, their existence has been predicted by the quark model [46]. Using the masses listed by the PDG and from the quark model, we find that the yield of these baryons is about $60 \%$ of the $\Lambda_{Q}$ yield in the thermal model and is thus appreciable. Including these higher resonances in the thermal model changes the baryon/meson ratios to

$$
\frac{\Lambda_{c}}{D^{0}} \simeq 0.27, \quad \frac{\Lambda_{b}}{\bar{B}^{0}} \simeq 0.86
$$

\section{THE COALESCENCE MODEL}

In the thermal model, the relative abundance of particles depends only on their masses. Whether diquarks exist in QGP thus does not affect the $\Lambda_{c} / D^{0}$ and $\Lambda_{b} / \bar{B}^{0}$ ratios in the thermal model. This is not the case in the quark coalescence model as to be shown in the rest of this paper.

\section{A. Formalism}

As in the coalescence model described in Refs. [13,47], which has been used in Ref. [48] to study the $D$ meson

\footnotetext{
${ }^{5}$ The decay of $B^{*}$ into $B$ is caused by electromagnetic interactions, $B^{*} \rightarrow B \gamma$, and can thus be distinguished in experiments.
}

transverse momentum spectrum in relativistic heavy ion collisions, we assume that quarks, antiquarks, and gluons in the produced QGP are uniformly distributed in a fire cylinder of volume $V$. Their momentum distributions are taken to be thermal in the transverse direction but uniform in midrapidity. For the Wigner functions of hadrons, they are expressed in terms of Gaussian functions. Modeling the effect of transverse radial flow of the fire cylinder by an effective temperature, the produced heavy meson transverse momentum spectrum is then given by

$$
\begin{aligned}
\frac{d N_{M}}{d \boldsymbol{p}_{M}}= & g_{M} \frac{(2 \sqrt{\pi} \sigma)^{3}}{V} \int d \boldsymbol{p}_{1} d \boldsymbol{p}_{2} \frac{d N_{1}}{d \boldsymbol{p}_{1}} \frac{d N_{2}}{d \boldsymbol{p}_{2}} \\
& \times \exp \left(-\boldsymbol{k}^{2} \sigma^{2}\right) \delta\left(\boldsymbol{p}_{M}-\boldsymbol{p}_{1}-\boldsymbol{p}_{2}\right),
\end{aligned}
$$

where $g_{M}$ is the statistical factor for colored quark and antiquark to form a color neutral meson, e.g., $g_{D^{0}}=1 / 36$ and $g_{D^{* 0}}=1 / 12$ for $D^{0}$ and $D^{* 0}$, respectively, and similarly for $\bar{B}^{0}$ and $\bar{B}^{* 0}$. The distribution of heavy quarks with transverse momentum $\boldsymbol{p}_{1}$ and light antiquarks with transverse momentum $\boldsymbol{p}_{2}$ in the fire cylinder frame are denoted by $d N_{1} / d \boldsymbol{p}_{1}$ and $d N_{2} / d \boldsymbol{p}_{2}$, respectively. The $\boldsymbol{p}_{M}$ is the transverse momentum of produced heavy meson. The relative transverse momentum $\boldsymbol{k}$ between the heavy quark and light antiquark is defined as

$$
\boldsymbol{k}=\frac{1}{m_{1}+m_{2}}\left(m_{2} \boldsymbol{p}_{1}^{\prime}-m_{1} \boldsymbol{p}_{2}^{\prime}\right),
$$

where $m_{1,2}$ are quark masses, and $\boldsymbol{p}_{1}^{\prime}$ and $\boldsymbol{p}_{2}^{\prime}$ are heavy quark and light antiquark transverse momenta, respectively, defined in the center-of-mass frame of produced heavy meson. The width parameter $\sigma$ is related to the harmonic oscillator frequency $\omega$ by $\sigma=1 / \sqrt{\mu \omega}$, where $\mu=m_{1} m_{2} /\left(m_{1}+m_{2}\right)$ is the reduced mass and can be related to the size of produced hadrons. Namely, the charge rms radius of produced meson is given by

$$
\left\langle r^{2}\right\rangle_{\mathrm{ch}}=\frac{3}{2} \frac{1}{\mu \omega} \frac{Q_{1} m_{2}^{2}+Q_{2} m_{1}^{2}}{\left(m_{1}+m_{2}\right)^{2}},
$$

with $Q_{i}$ being the charge of the $i$ th (anti)quark. To reproduce the root-mean-square charge radii $0.43 \mathrm{fm}$ of $D^{+}$and $0.62 \mathrm{fm}$ of $\mathrm{B}^{+}$as predicted by the light-front quark model of Ref. [49], an oscillator frequency $\omega=0.33 \mathrm{GeV}$ is needed.

We note that contrary to that for mesons consisting of only light quarks, the coalescence formula, Eq. (18), does not require heavy and light quarks to have similar momenta to form heavy hadrons. Instead, quarks of similar velocities have the most probable chance to form hadrons.

Similarly, the coalescence formula for forming $\Lambda_{Q}, \Sigma_{Q}$, $\Xi_{Q}, \Xi_{Q}^{\prime}$, and $\Omega_{Q}$ as well as their resonances from three quarks are given by

$$
\begin{aligned}
\frac{d N_{B}}{d \boldsymbol{p}_{B}}= & g_{B} \frac{(2 \sqrt{\pi})^{6}\left(\sigma_{1} \sigma_{2}\right)^{3}}{V^{2}} \int d \boldsymbol{p}_{1} d \boldsymbol{p}_{2} d \boldsymbol{p}_{3} \frac{d N_{1}}{d \boldsymbol{p}_{1}} \frac{d N_{2}}{d \boldsymbol{p}_{2}} \frac{d N_{3}}{d \boldsymbol{p}_{3}} \\
& \times \exp \left(-\boldsymbol{k}_{1}^{2} \sigma_{1}^{2}-\boldsymbol{k}_{2}^{2} \sigma_{2}^{2}\right) \delta\left(\boldsymbol{p}_{B}-\boldsymbol{p}_{1}-\boldsymbol{p}_{2}-\boldsymbol{p}_{3}\right),
\end{aligned}
$$

where the index 3 refers to the heavy quark and indices 1 and 2 to light quarks, and the statistical factor $g_{B}$ has values $1 / 108,1 / 36$, and $1 / 18$ for $\Lambda_{Q}, \Sigma_{Q}$, and $\Sigma_{Q}^{*}$, respectively, and 
$1 / 54,1 / 18,1 / 108$, and $1 / 36$ for $\Xi_{Q}\left(\Xi_{Q}^{\prime}\right), \Xi_{Q}^{*}, \Omega_{Q}$, and $\Omega_{Q}^{*}$, respectively. The relative transverse momenta defined in the center of mass frame of produced baryon are

$$
\begin{aligned}
\boldsymbol{k}_{1} & =\frac{1}{m_{1}+m_{2}}\left(m_{2} \boldsymbol{p}_{1}^{\prime}-m_{1} \boldsymbol{p}_{2}^{\prime}\right) \\
\boldsymbol{k}_{2} & =\frac{1}{m_{1}+m_{2}+m_{3}}\left[m_{3}\left(\boldsymbol{p}_{1}^{\prime}+\boldsymbol{p}_{2}^{\prime}\right)-\left(m_{1}+m_{2}\right) \boldsymbol{p}_{3}^{\prime}\right]
\end{aligned}
$$

The width parameters $\sigma_{i}$ are $\sigma_{i}=1 / \sqrt{\mu_{i} \omega}$, where

$$
\mu_{1}=\frac{m_{1} m_{2}}{m_{1}+m_{2}}, \quad \mu_{2}=\frac{\left(m_{1}+m_{2}\right) m_{3}}{m_{1}+m_{2}+m_{3}},
$$

and the oscillator frequency $\omega$ is related to the baryon charge radius by

$$
\begin{aligned}
\left\langle r^{2}\right\rangle_{\mathrm{ch}}= & \frac{3}{2 \omega} \frac{1}{m_{1}+m_{2}+m_{3}} \\
& \times\left(\frac{m_{2}+m_{3}}{m_{1}} Q_{1}+\frac{m_{3}+m_{1}}{m_{2}} Q_{2}+\frac{m_{1}+m_{2}}{m_{3}} Q_{3}\right) .
\end{aligned}
$$

The root-mean-square charge radii of $\Lambda_{c}$ and $\Lambda_{b}$ predicted by the quark model have similar values of about $0.39 \mathrm{fm}$ [50], and they are reproduced by the oscillator frequencies $0.43 \mathrm{GeV}$ and $0.41 \mathrm{GeV}$, respectively. These values are $25-30 \%$ larger than the ones for heavy mesons. As we shall show in the next subsection, to convert as many heavy quarks of small $p_{T}$ into heavy hadrons via coalescence as possible ${ }^{6}$ requires, however, smaller oscillator frequencies or larger heavy hadron radii.

For production of $\Lambda_{Q}$ baryons from the coalescence of heavy quarks and $[u d]$ diquarks, we consider $\Lambda_{Q}$ baryons as made of a heavy quark and a light diquark only. Their spectrum can thus be obtained from Eq. (18) by replacing $g_{M}$ with $g_{B}^{\prime}=1 / 9$. Explicitly, it is written as

$$
\begin{aligned}
\frac{d N_{B}}{d \boldsymbol{p}_{B}}= & g_{B}^{\prime} \frac{\left(2 \sqrt{\pi} \sigma_{d q}\right)^{3}}{V} \int d \boldsymbol{p}_{1} d \boldsymbol{p}_{2} \frac{d N_{1}}{d \boldsymbol{p}_{1}} \frac{d N_{2}}{d \boldsymbol{p}_{2}} \\
& \times \exp \left(-\boldsymbol{k}^{2} \sigma_{d q}^{2}\right) \delta\left(\boldsymbol{p}_{B}-\boldsymbol{p}_{1}-\boldsymbol{p}_{2}\right) .
\end{aligned}
$$

The width parameter $\sigma_{d q}$ or corresponding oscillator frequency $\omega_{d q}$ is determined by fitting the sizes of heavy baryons used in the three-quark model, i.e., $\omega=0.25 \mathrm{GeV}$ and $0.26 \mathrm{GeV}$ for $\Lambda_{c}$ and $\Lambda_{b}$, respectively.

The scalar diquarks can also lead to formation of excited states of $\Lambda_{Q}$ baryons with $j^{\pi}=\frac{1}{2}^{-}$and $\frac{3}{2}^{-}$, which can decay into $\Lambda_{Q}$ by emitting pions. We note that the mass differences between $j^{\pi}=\frac{1}{2}^{-}$and $\frac{3}{2}^{-}$states are small because of heavy quark spin symmetry, e.g., the lowest excited states of $\Lambda_{c}$ are $\Lambda$ (2593) of $j^{\pi}=\frac{1}{2}^{-}$and $\Lambda_{c}$ (2625) of $j^{\pi}=\frac{3}{2}^{-}$, and thus have a mass difference of only $32 \mathrm{MeV}$. Although the number of these resonances is rather small in the thermal model due to their higher masses compared to those of ground state $\Lambda_{Q}$ baryons, it is not the case in the coalescence model if diquarks

\footnotetext{
${ }^{6}$ This is similar to the assumption introduced in the thermal model that all charmed quarks are converted into heavy hadrons.
}

exist in QGP. Since excited $\Lambda_{Q}$ baryons have negative parities, the scalar diquark inside them is in a relative $P$-wave state with respect to the heavy quark. The coalescence formula for the production of such hadrons is

$$
\begin{aligned}
\frac{d N_{B}}{d \boldsymbol{p}_{B}}= & g_{B}^{\prime} \frac{2\left(2 \sqrt{\pi} \sigma_{d q}\right)^{3}}{3 V} \int d \boldsymbol{p}_{1} d \boldsymbol{p}_{2} \frac{d N_{1}}{d \boldsymbol{p}_{1}} \frac{d N_{2}}{d \boldsymbol{p}_{2}} \sigma_{d q}^{2} k^{2} \\
& \times \exp \left(-\boldsymbol{k}^{2} \sigma_{d q}^{2}\right) \delta\left(\boldsymbol{p}_{B}-\boldsymbol{p}_{1}-\boldsymbol{p}_{2}\right),
\end{aligned}
$$

using the Wigner function given in Refs. [51,52]. In the above, $k$ is similarly defined as in Eq. (19) and the width parameter $\sigma_{d q}$ is taken to have same value as that for the ground state $\Lambda_{Q}$.

\section{B. Quark distribution functions in QGP}

For the quark distribution functions in quark-gluon plasma, we use the thermal distribution for light quarks, namely,

$$
\frac{d N_{q}}{d \boldsymbol{p}}=\lambda_{q} \frac{g_{q} V}{(2 \pi)^{3}} m_{T} \exp \left(-m_{T} / T_{\mathrm{eff}}\right),
$$

where $g_{q}=6, m_{T}=\sqrt{\boldsymbol{p}^{2}+m_{q}^{2}}$, and $\boldsymbol{p}$ is the transverse momentum. Instead of the local temperature and transverse flow velocity, we use an effective temperature $T_{\text {eff }}(=200 \mathrm{MeV})$ and introduce a normalization factor $\lambda_{q}$ to fix the total number of quarks as in Ref. [53]. Following Ref. [54], we assume that the phase transition temperature is $T_{C}=175 \mathrm{MeV}$ and the volume of the quark-gluon plasma formed in rapidity $|y| \leqslant 0.5$ is $V=1,000 \mathrm{fm}^{3}$ for central $\mathrm{Au}+\mathrm{Au}$ collisions at $\sqrt{s_{N N}}=200 \mathrm{GeV}$ at RHIC. This gives the light quark numbers $N_{u}=N_{d} \approx 245$ and the strange quark number $N_{s} \approx 149$ if the constituent light quark and strange quark masses are taken to be $300 \mathrm{MeV}$ and $475 \mathrm{MeV}$, respectively. The resulting normalization factors $\lambda_{q}$ for light and strange quarks are then 0.95 and 0.81 , respectively.

Diquarks are also assumed to be in thermal equilibrium and their distribution $d N_{[u d]} / d \boldsymbol{p}$ is then the same as given in Eq. (27) with $g_{[u d]}=3$. The number of diquarks depends on the diquark mass and is in the range of $77 \sim 44$ with $m_{[u d]}=455 \sim 600 \mathrm{MeV}$ [20]. If diquarks exist in QGP, the number of free light quarks is reduced by twice the number of diquarks so that the total number of light quarks is preserved. This would cause a reduction of meson yields and an enhancement of baryon yields and thus induces larger $\Lambda_{Q} / H^{0}$ ratios. Results to be presented below are obtained by using the diquark mass $m_{[u d]}=455 \mathrm{MeV}$, which is in the range of diquark mass estimated in Ref. [55]. The sensitivity of these results to the diquark mass will, however, be briefly discussed.

For heavy quark transverse momentum distributions in midrapidity, we adopt those parameterized in Ref. [8] for central $\mathrm{Au}+\mathrm{Au}$ collisions at $\sqrt{s_{N N}}=200 \mathrm{GeV}$ at RHIC, i.e.,

$$
\begin{aligned}
\frac{d N_{c}}{d \boldsymbol{p}} & =\frac{19.2\left[1+(p / 6)^{2}\right]}{(1+p / 3.7)^{12}[1+\exp (0.9-2 p)]}, \\
\frac{d N_{b}}{d \boldsymbol{p}} & =0.0025\left[1+\left(\frac{p}{16}\right)^{5}\right] \exp (-p / 1.495),
\end{aligned}
$$

with the transverse momentum $p$ in units of GeV. These distributions were obtained from heavy quark $p_{T}$ spectra from 
$p p$ collisions at same energy by the number of binary collisions ( $\sim 960)$ in $\mathrm{Au}+\mathrm{Au}$ collisions. For bottom quarks, it was taken from the upper limit of the uncertainty band of the pQCD predictions in Ref. [56] for $p p$ collisions, as no experimental measurements are available at RHIC. The charm quark $p_{T}$ spectrum in $p p$ collisions is, on the other hand, determined from fitting simultaneously measured $p_{T}$ spectrum of charmed mesons from $d+$ Au collisions [57] and of electrons from heavy meson decays in $p p$ collisions.

Including heavy quark energy loss as estimated in Ref. [8], the resulting heavy quark transverse momentum distribution in midrapidity can be parametrized as

$$
\frac{d N_{Q}^{\mathrm{EL}}}{d \boldsymbol{p}}=\frac{d N_{Q}}{d \boldsymbol{p}} L_{Q}(p),
$$

with

$$
\begin{aligned}
& L_{c}=0.8 \exp (-p / 1.2)+0.6 \exp (-p / 15), \\
& L_{b}=0.36+0.84 \exp (-p / 10),
\end{aligned}
$$

for charm and bottom quarks, respectively, and $p$ again in $\mathrm{GeV}$. The heavy quark numbers are then estimated to be $N_{c} \simeq$ 9.23 and $N_{b} \simeq 0.035$. The charm quark number used here is about a factor three larger than that used in Ref. [20] based on perturbative QCD calculations. This, however, does not affect the $\Lambda_{Q} / H^{0}$ ratios. For the heavy quark masses, we use $m_{c}=1.5 \mathrm{GeV}$ and $m_{b}=5.0 \mathrm{GeV}$.

\section{Results}

To calculate the spectra of $H^{0}$ and $\Lambda_{Q}$ produced in relativistic heavy ion collisions, we consider two scenarios for $\Lambda_{Q}$ production. In the first model, we assume that the $\Lambda_{Q}$ baryons have no diquark structure and there are no diquarks in QGP either. Therefore, $\Lambda_{Q}, \Sigma_{Q}, \Sigma_{Q}^{*}, \Xi_{Q}, \Xi_{Q}^{\prime}, \Xi_{Q}^{*}, \Omega_{Q}$, and $\Omega_{Q}^{*}$ baryons are all formed by three-quark coalescence. We further include heavy mesons that consist of strange quarks such as $D_{s}$ and $B_{s}$ and their resonances. Using the same oscillator frequency for both heavy mesons and baryons as shown approximately in the quark model, we adjust its value to convert all heavy quarks of small $p_{T}$ into heavy hadrons via coalescence as in the thermal model. This leads to $\omega=0.106 \mathrm{GeV}$ for charmed hadrons and $\omega=0.059 \mathrm{GeV}$ for bottom hadrons. The resulting $D^{+}$and $B^{+}$charge radii are $0.74 \mathrm{fm}$ and $1.44 \mathrm{fm}$, respectively, which are factors of 1.7 and 2.3 larger than those predicted by the light-front quark model of Ref. [49]. The required smaller oscillator frequencies may be partly due to the change in hadron radii in medium [58] and partly due to the incomplete treatment of production and decay of hadron resonances in the present study.

The second model assumes the diquark structure in $\Lambda_{Q}$ and the existence of diquarks in QGP as in Ref. [20]. In this case, $\Lambda_{Q}$ baryons are formed by diquark-heavy-quark coalescence in addition to three-quark coalescence. ${ }^{7}$ Together with the

\footnotetext{
${ }^{7}$ Strictly speaking, the three quarks should coalesce to form the $\Lambda_{Q}$ in diquark structure. Here we simply approximate this production process by three-quark coalescence to form non-diquark $\Lambda_{Q}$. This
}

$\Sigma_{Q}$ and $\Sigma_{Q}^{*}$ resonance contributions as well as those from $\Xi_{Q}, \Xi_{Q}^{\prime}, \Xi_{Q}^{*}, \Omega_{Q}$, and $\Omega_{Q}^{*}$ resonances, which are formed only from three-quark coalescence as they do not contain the scalar diquark structure, excited $\Lambda_{Q}\left(\frac{1}{2}^{-}\right)$and $\Lambda_{Q}\left(\frac{3}{2}^{-}\right)$baryons are also included using the $P$-wave diquark-heavy-quark coalescence. ${ }^{8}$ As in the three-quark coalescence model, the oscillator frequencies or width parameters are determined by requiring that all low $p_{T}$ heavy quarks are converted to hadrons. Besides the common oscillator frequency $\omega$ for mesons and three-quark baryons, the additional oscillator frequency for the heavy-quark and diquark system is fixed by reproducing the same $\Lambda_{Q}$ radius used in the three-quark configuration. Then we have $\omega=0.09 \mathrm{GeV}$ for charmed hadrons and $\omega=0.049 \mathrm{GeV}$ for bottom hadrons, leading to $0.81 \mathrm{fm}$ and $1.58 \mathrm{fm}$ for the $\mathrm{D}^{+}$and $\mathrm{B}^{+}$charged radii, respectively. These values lead to $\omega_{d q}=0.053 \mathrm{GeV}$ and $0.029 \mathrm{GeV}$, respectively, for charmed and bottom baryons consisting of diquarks. In obtaining the contribution of resonances to the ground state $H^{0}$ and $\Lambda_{Q}$ spectra, we neglect for simplicity the small momentum shift in their decays.

In both models, remaining heavy quarks that are not converted to hadrons via coalescence, which mostly have high $p_{T}$, are converted to heavy hadrons by fragmentation. This is achieved by assuming that a heavy quark of transverse momentum $p_{T}$ fragments into $\Lambda_{Q}$ and $H^{0}$ of same momentum and with a ratio similar to that given by the PYTHIA model, i.e.,

$$
\begin{aligned}
& \left(\frac{\Lambda_{c}}{D^{0}}\right)_{\mathrm{fr}}=0.05 \exp \left[-\left(p_{T}-4.0\right)^{2} / 8.0\right]+0.1, \\
& \left(\frac{\Lambda_{b}}{\bar{B}^{0}}\right)_{\mathrm{fr}}=0.75,
\end{aligned}
$$

with $p_{T}$ in units of $\mathrm{GeV}$.

\section{Relative production fractions of heavy hadrons and $\Lambda_{Q} / H^{0}$ ratios}

Our results for the relative production fractions of heavy hadrons produced in central $\mathrm{Au}+\mathrm{Au}$ collisions at $\sqrt{s_{N N}}=$ $200 \mathrm{GeV}$ are presented in Table I together with those from the PYTHIA model and the thermal model. It is seen that the relative production fraction of $D^{0}$ among all produced charmed hadrons in the three-quark coalescence model is 0.35 , while it is 0.44 and 0.61 for the thermal model and the PYTHIA model, respectively. This fraction reduces to 0.28 in the diquark model. For $\bar{B}^{0}$, its relative production fractions among all produced bottom hadrons are 0.101, 0.097, 0.052, and 0.043 in the PYTHIA model, the thermal model, the three-quark model, and the diquark model, respectively.

thus gives an upper bound for the yield of $\Lambda_{c}$ from three independent quarks in QGP as pointed out in Ref. [20].

${ }^{8}$ In general, coalescence of three independent quarks can also form negative parity baryons. Since this process has a smaller contribution than that due to the production of positive parity baryons [59], it is not considered in present work. 
TABLE I. Relative production fractions of midrapidity heavy hadrons produced in central Au + Au collisions at $\sqrt{s_{N N}}=200 \mathrm{GeV}$. The heavy-strange baryon $\left(\Xi_{Q}, \Xi_{Q}^{\prime}\right.$, and $\left.\Omega_{Q}\right)$ yield is suppressed in the PYTHIA model and thus neglected in this work.

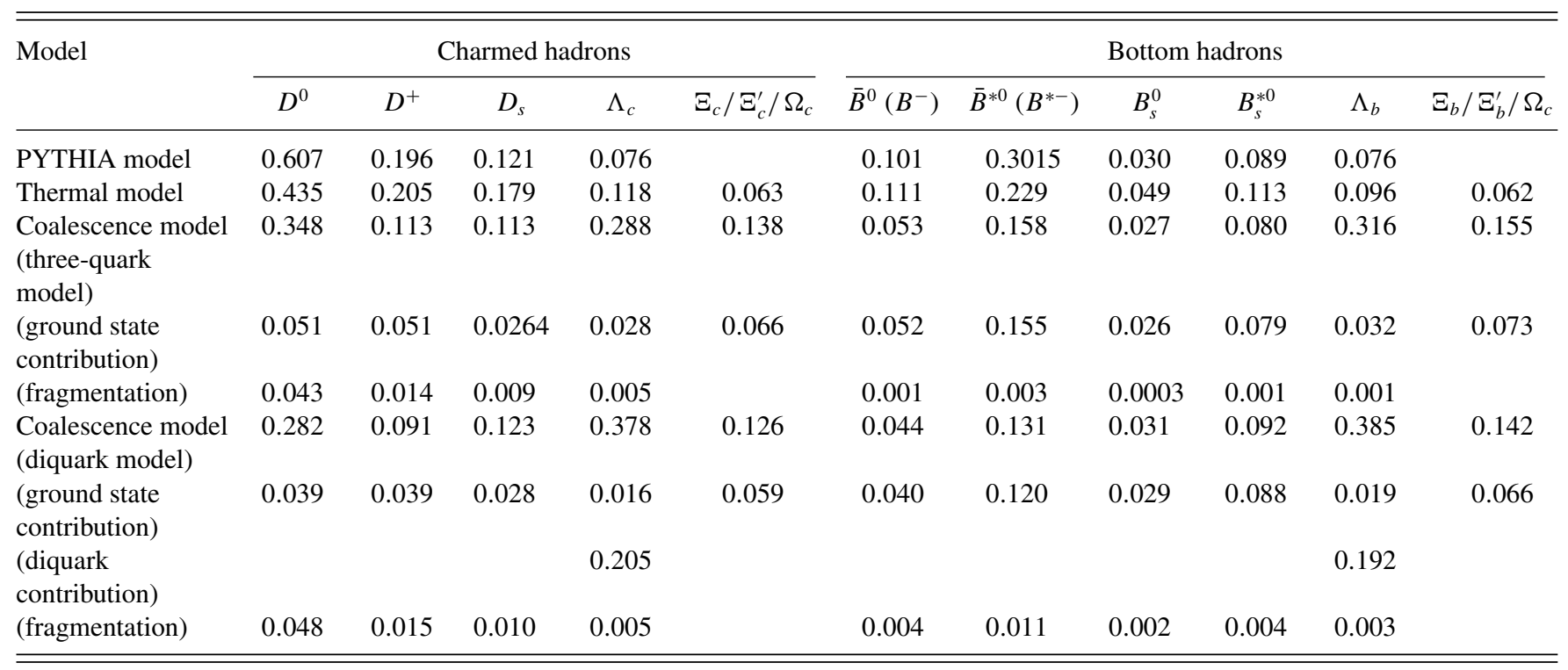

For the $\Lambda_{Q} / H^{0}$ ratios, we find that without resonance contribution $\Lambda_{c} / D^{0}=0.55$ in the three-quark model and $\Lambda_{c} / D^{0}=2.2$ in the diquark model. These results are different from those of Ref. [20], namely, the $\Lambda_{c} / D^{0}$ is larger and the enhancement of this ratio due to the presence of diquarks is about 4 while it was about 8 in Ref. [20]. These differences come from the different values for the oscillator frequencies or width parameters as well as the fact that in Ref. [20] the number of free quarks in the quark-gluon plasma is not reduced by the presence of diquarks. For bottom hadrons, we obtain $\Lambda_{b} / \bar{B}^{0}=0.6$ in the three-quark model, which increases by a factor of 3.5 to $\Lambda_{b} / \bar{B}^{0}=2.1$ in the diquark model.

Including resonances enhances both $\Lambda_{Q}$ and $H^{0}$ production. We find that in the three-quark model, about $70 \%$ of $D^{0}$ and about $90 \%$ of $\Lambda_{Q}$ are produced through resonance decays. The importance of resonances is also seen in the diquark model. In particular, the diquark contribution through negative parity $\Lambda_{Q}$ resonances is non-negligible. The number of negative parity $\Lambda_{Q}$ resonance of spin-1/2 turns out to be about half of the ground state $\Lambda_{Q}$ baryon number. Since $\Lambda_{Q}\left(\frac{1}{2}^{-}\right)$and $\Lambda_{Q}\left(\frac{3}{2}^{-}\right)$resonances are almost degenerate in mass, the number of negative parity $\Lambda_{Q}$ resonances is about two times that of the ground state $\Lambda_{Q}$ after taking into account the spin degeneracy. This should be contrasted with the thermal model which predicts suppressed yields of negative parity $\Lambda_{Q}$ resonances due to their large masses. As a result, we find that about $70 \%$ of $D^{0}$ and about $75 \%$ of $\Lambda_{Q}$ are produced through resonance decays in the diquark model.

The contribution from heavy quark fragmentation is small, particularly to the ratios of particle numbers. This is because we have assumed that most hadrons are produced by coalescence of low $p_{T}$ heavy quarks. The fragmentation contribution is thus non-negligible only for high $p_{T}$ hadrons, which constitute less than $8 \%$ of produced total heavy hadrons.
The final $\Lambda_{Q} / H^{0}$ ratios after including fragmentation and resonance contributions are

$$
\Lambda_{c} / D^{0}=0.83, \quad \Lambda_{b} / \bar{B}^{0}=6.00,
$$

for the three-quark model, and

$$
\Lambda_{c} / D^{0}=1.34, \quad \Lambda_{b} / \bar{B}^{0}=8.79,
$$

for the diquark model. Therefore, the enhancement factor for these ratios due to diquarks in QGP is about 1.6 for both charmed and bottom hadrons.

Our results thus indicate that the $\Lambda_{c} / D^{0}\left(\Lambda_{b} / \bar{B}^{0}\right)$ ratio in heavy ion collisions predicted by the coalescence model is about a factor of 6.4 (8.6) larger than the predictions of the PYTHIA model in Sec. II and about a factor of 3.1 (7.0) larger than that from the thermal model in Sec. III. The existence of diquarks in QGP further enhances this factor to 10.3 (12.6) and 5.0 (10.2), compared to those from the PYTHIA and thermal models, respectively.

\section{Transverse momentum spectra of heavy hadrons}

The transverse momentum spectra of $\Lambda_{Q}$ and $H^{0}$ are shown in Figs. 1 and 2 for charmed and bottom hadrons, respectively, together with the transverse momentum dependence of the $\Lambda_{Q} / H^{0}$ ratio. Solid lines are results for the three-quark model and dashed lines are those for the diquark model. Compared with the PYTHIA results shown by filled circles, the enhancement of $\Lambda_{Q}$ yield and the suppression of $H^{0}$ yield are evident. The bottom hadron spectra are seen to be harder than the charmed hadron spectra due to the flatter bottom quark spectrum than the charm quark spectrum.

Compared with measured $\bar{p} / \pi^{-}$and $\Lambda / K_{S}^{0}$ ratios [60-62], the obtained $\Lambda_{Q} / H^{0}$ ratios show very different behavior. Although the $\Lambda_{c} / D^{0}$ ratio has a similar shape as the $\bar{p} / \pi^{-}$ and $\Lambda / K_{S}^{0}$ ratios, which could be approximated by a Gaussian 


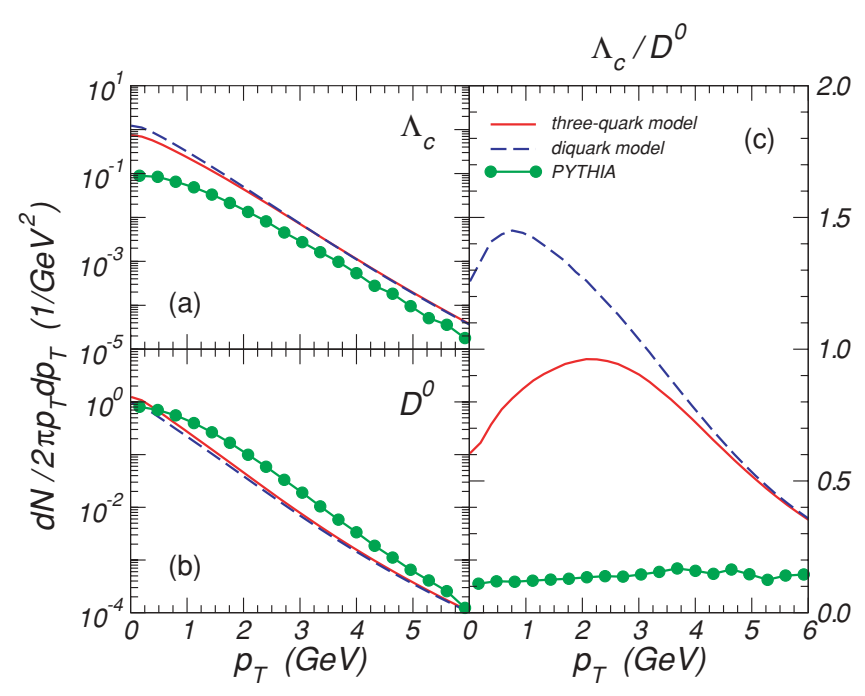

FIG. 1. (Color online) Spectra of (a) $\Lambda_{c}$ and (b) $D^{0}$, and (c) the ratio $\Lambda_{c} / D^{0}$ in midrapidity $(|y| \leqslant 0.5)$ for central $\mathrm{Au}+\mathrm{Au}$ collisions at $\sqrt{s_{N N}}=200 \mathrm{GeV}$. Solid lines are for the three-quark model and dashed lines are for the diquark model. Results from the PYTHIA model are shown by filled circles.

shape as assumed in Ref. [12], it has a much larger width and is thus much flatter than the light baryon/meson ratios. Furthermore, the $\Lambda_{b} / \bar{B}^{0}$ ratio is even flatter than the $\Lambda_{c} / D^{0}$ ratio. This behavior can also be found in the spectra of $\Lambda_{Q}$ and $H^{0}$. Namely, while the spectra of $\Lambda_{b}$ and $\bar{B}^{0}$ have very similar $p_{T}$ dependence (Fig. 2), those of $\Lambda_{c}$ and $D^{0}$ show somewhat different $p_{T}$ dependence (Fig. 1). All these behaviors originate from the large mass of heavy quarks. In the infinite mass limit, the momentum of a heavy hadron is completely carried by the heavy quark, and the role of light quarks or diquarks is just to give different production probability depending on the heavy hadron wave function. Therefore, the $\Lambda_{Q} / H^{0}$ ratio in the infinite heavy mass limit becomes a constant and shows no momentum dependence. In the case of the $\Lambda_{c} / D^{0}$ ratio,

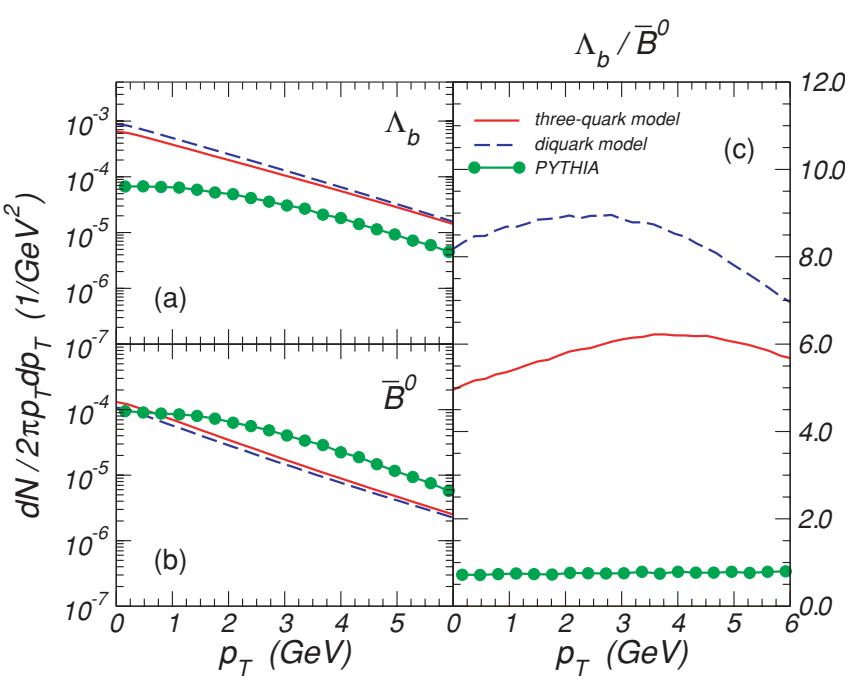

FIG. 2. (Color online) Same as Fig. 1 for (a) $\Lambda_{b}$ spectrum, (b) $\bar{B}^{0}$ spectrum, and (c) the $\Lambda_{b} / \bar{B}^{0}$ ratio. finite mass effects are not negligible but its dependence on the transverse momentum is less strong than in the $\Lambda / K_{S}^{0}$ ratio. This is clearly seen in $p p$ collisions as shown by the PYTHIA model results in Figs. 1 and 2. Although the coalescence model shows a $p_{T}$ dependence of the $\Lambda_{Q} / H^{0}$ ratio, this dependence weakens as the heavy quark mass increases.

We also find that inclusion of diquarks in QGP causes a shift of the peak positions in the $\Lambda_{Q} / H^{0}$ ratios to lower values of $p_{T}$. In the three-quark model, the $\Lambda_{c} / D^{0}$ ratio peaks at $p_{T} \simeq 2 \mathrm{GeV}$, which shifts to $p_{T} \simeq 0.8 \mathrm{GeV}$ in the diquark model. The change of the peak position is caused by both the reduction of the $D^{0}$ meson spectrum and the enhancement of the $\Lambda_{c}$ spectrum in the diquark model. We find, however, that the latter has a larger effect on the peak position in the $\Lambda_{c} / D^{0}$ ratio. Since the enhancement of $\Lambda_{c}$ due to light diquarks is more appreciable in the low $p_{T}$ region, the peak of the $\Lambda_{c} / D^{0}$ ratio in the diquark model thus appears at a lower value of $p_{T}$ compared to that in the three-quark model. The $\Lambda_{b} / \bar{B}^{0}$ ratio also shows a shift in the peak position, i.e., from $p_{T} \simeq 3.5 \mathrm{GeV}$ in the three-quark model to $2 \mathrm{GeV}$ in the diquark model, but it is still much flatter than the $\Lambda_{c} / D^{0}$ ratio.

For a more realistic momentum distribution of heavy hadrons in relativistic heavy ion collisions after their production from QGP, their production and annihilation due to collisions of and with surrounding particles should be investigated by a transport model like the AMPT model [63,64]. However, cross sections for reactions involving open charmed hadrons are small [65,66], and it has been shown in Ref. [51] that the effects of multiple scattering would be small with such small cross sections. Since this work is at the level of an exploratory study, such an elaborated investigation is not yet called for.

\section{CONCLUSIONS AND DISCUSSIONS}

In this paper, we have studied the $\Lambda_{Q} / H^{0}\left(\Lambda_{c} / D^{0}\right.$ and $\Lambda_{b} / \bar{B}^{0}$ ) ratios in heavy ion collisions, particularly the enhancement of these ratios due to coalescence of heavy quarks with light quarks as well as diquarks that might exist in the produced QGP. We have also considered the resonance decay effects which are shown to be important for understanding the particle production ratios in $p p$ collisions. Our simple estimate based on the thermal model as well as predictions of more sophisticated statistical models [42,43] show that resonance decays also plays a crucial role for heavy hadron production in heavy ion collisions. We have, therefore, included the contribution from resonances in estimating these ratios.

We have determined the width parameters of hadron Wigner functions used in the coalescence model by requiring that the majority of heavy hadrons at low $p_{T}$ are formed from coalescence, which is similar to that assumed in the thermal model. The remaining heavy quarks are then converted to heavy hadrons via fragmentation as in $p p$ collisions. The resulting $\Lambda_{c} / D^{0}$ and $\Lambda_{b} / \bar{B}^{0}$ ratios in central $\mathrm{Au}+\mathrm{Au}$ collisions at $\sqrt{s_{N N}}=200 \mathrm{GeV}$ are found to be 1.34 and 8.79, respectively, which are about 10.3 and 12.6 larger than the predictions of the PYTHIA model, and about a factor of 5.0 and 
10.2 larger than those of the thermal model. The enhancement of the $\Lambda_{Q} / H^{0}$ ratio due to diquarks is, however, only about 1.6 for both charmed and bottom hadrons, which is smaller than that predicted in Ref. [20]. The difference mainly arises from the different constraints used here and in Ref. [20] for determining the oscillator frequencies or width parameters in the hadron Wigner functions and from maintaining the same total light quark numbers in both the three-quark model and diquark model in the present study.

The enhancement due to diquarks would have been larger if we do not take into account the resonance contribution. This is because of the enhancement of $\Lambda_{Q}$ baryons from $\Sigma_{Q}$ and $\Sigma_{Q}^{*}$ decays, whose production is not affected by the presence of diquarks in QGP. This is different from the production of strange $\Lambda$ to which $\Sigma$ can not decay by strong interactions. Furthermore, including the resonance contribution increases the $\Lambda_{b} / \bar{B}^{0}$ ratio much more than the $\Lambda_{c} / D^{0}$ ratio because $B^{*}$ cannot decay into $B$ by strong interactions as a result of heavy quark spin symmetry. Finally, this model leads to the reduction of $D^{0}$ and $\bar{B}^{0}$ mesons by factors of about 2.2 compared to the PYTHIA model.

Contrary to the PYTHIA model, we have found that the yields of heavy baryons with strange quark(s) are not small. Their yield was estimated to be about $60 \%$ of the $\Lambda_{Q}$ yield in the thermal model. In the coalescence model, this is about $50 \%$ and $35 \%$ of the $\Lambda_{Q}$ yield in the three-quark model and diquark model, respectively.

We have also compared the transverse momentum dependence of the $\Lambda_{Q} / H^{0}$ ratios with that of measured $\Lambda / K_{S}^{0}$ ratio and found that the $\Lambda_{Q} / H^{0}$ ratios have a weaker dependence on the transverse momentum because of the massive quarks inside heavy hadrons. In particular, this leads to a very weak dependence of the $\Lambda_{b} / \bar{B}^{0}$ ratio on the transverse momentum.

We have further found that the $\Lambda_{Q} / D^{0}$ ratio peaks at $p_{T} \simeq$ $2 \mathrm{GeV}$ in the three-quark model and at $p_{T} \simeq 0.8 \mathrm{GeV}$ in the diquark model. Therefore, the enhancement of heavy baryon production over heavy meson production due to diquarks can mostly be observed at low $p_{T}$ region. We have also estimated that the ratio $\Lambda_{c} / \Sigma_{c}^{0} \simeq 23$, which is less than that of Ref. [23] by a factor of $\sim 4$.

These results are based on a diquark mass of $445 \mathrm{MeV}$ or a binding energy of $145 \mathrm{MeV}$. Because of the thermal factor, the number of diquarks decreases as the diquark mass increases. This would reduce both $\Lambda_{c}$ and $\Lambda_{b}$ production and increase that of $D^{0}$ and $\bar{B}^{0}$, reducing thus the $\Lambda_{Q} / H^{0}$ ratios. For example, if the diquark mass is $550 \mathrm{MeV}$, the enhancement of the $\Lambda_{c} / D^{0}$ ratio due to diquarks would reduce from 1.6 to 1.3. The peak position in the ratio changes, however, very little.

In studying resonance production in the coalescence model, we have assumed that the effect due to energy mismatch between quarks and produced hadron is small. Since the coalescence model can be viewed as formation of bound states from interacting particles in the system with energy mismatch balanced by other particles in the system, this would be reasonable if the energy mismatch is small. Otherwise, the correction factor can, in principle, be determined by evaluating the transition probability in the presence of other particles. Also, the coalescence model can be further improved by imposing energy conservation using Breit-Wigner type spectral functions for the produced hadrons [67]. This would naturally lead to suppressed production rate due to the energy mismatch between quarks and produced hadron. Improving the coalescence model, therefore, deserves further studies.

One may also consider contributions to heavy hadron production from other diquark states. It is well-known that the color-spin interaction for color-sextet diquark of spin-1 is, although not strong, attractive. (See, e.g., Ref. [33].) This diquark, of course, cannot form a color-singlet baryon with one heavy quark, and color-sextet diquarks are thus disfavored in phenomenological models [31]. However, colorsextet diquarks in QGP can produce color-singlet baryons by first forming color-octet or color-decuplet baryons and then neutralizing their colors by interacting with quarks and gluons in QGP. Therefore, if we assume the existence of (weakly-bound) color-sextet vector diquarks in QGP, it may bring in additional enhancement of $\Lambda_{Q}$ baryon production. Since this mechanism is very model-dependent, the existence and properties of such diquark states in QGP should be understood before estimating their contributions to heavy hadron production.

\section{ACKNOWLEDGMENTS}

We would like to thank Zi-Wei Lin for help with the HIJING/PYTHIA simulations. This work was supported by the U.S. National Science Foundation under Grant Nos. PHY0457265 and PHY-0758115, the Welch Foundation under Grant No. A-1358, and the Korea Research Foundation under Grant No. KRF-2006-C00011.
[1] A. Adare et al. (PHENIX Collaboration), Phys. Rev. Lett. 98, 172301 (2007).

[2] B. I. Abelev et al. (STAR Collaboration), Phys. Rev. Lett. 98, 192301 (2007).

[3] Yu. L. Dokshitzer and D. E. Kharzeev, Phys. Lett. B519, 199 (2001).

[4] M. Djordjevic and M. Gyulassy, Nucl. Phys. A733, 265 (2004).

[5] H. van Hees and R. Rapp, Phys. Rev. C 71, 034907 (2005).

[6] M. Djordjevic, M. Gyulassy, R. Vogt, and S. Wicks, Phys. Lett. B632, 81 (2006).

[7] H. van Hees, V. Greco, and R. Rapp, Phys. Rev. C 73, 034913 (2006).
[8] C. M. Ko and W. Liu, Nucl. Phys. A783, 233c (2007).

[9] B. Zhang, L.-W. Chen, and C.-M. Ko, Phys. Rev. C 72, 024906 (2005).

[10] P. B. Gossiaux and J. Aichelin, Phys. Rev. C 78, 014904 (2008).

[11] P. Sorensen and X. Dong, Phys. Rev. C 74, 024902 (2006); P. Sorensen, Eur. Phys. J. C 49, 379 (2007).

[12] G. Martínez-García, S. Gadrat, and P. Crochet, Phys. Lett. B663, 55 (2008).

[13] V. Greco, C. M. Ko, and P. Lévai, Phys. Rev. Lett. 90, 202302 (2003).

[14] V. Greco, C. M. Ko, and P. Lévai, Phys. Rev. C 68, 034904 (2003). 
[15] R. J. Fries, B. Müller, C. Nonaka, and S. A. Bass, Phys. Rev. Lett. 90, 202303 (2003).

[16] D. Kharzeev, Phys. Lett. B378, 238 (1996).

[17] S. E. Vance, M. Gyulassy, and X.-N. Wang, Phys. Lett. B443, 45 (1998).

[18] S. E. Vance and M. Gyulassy, Phys. Rev. Lett. 83, 1735 (1999).

[19] V. Topor Pop, M. Gyulassy, J. Barrette, and C. Gale, Phys. Rev. C 72, 054901 (2005)

[20] S. H. Lee, K. Ohnishi, S. Yasui, I.-K. Yoo, and C. M. Ko, Phys. Rev. Lett. 100, 222301 (2008)

[21] E. V. Shuryak and I. Zahed, Phys. Rev. C 70, 021901(R) (2004); Phys. Rev. D 70, 054507 (2004).

[22] J. F. Donoghue and K. S. Sateesh, Phys. Rev. D 38, 360 (1988).

[23] K. S. Sateesh, Phys. Rev. D 45, 866 (1992).

[24] M. Ida and R. Kobayashi, Prog. Theor. Phys. 36, 846 (1966).

[25] D. B. Lichtenberg and L. J. Tassie, Phys. Rev. 155, 1601 (1967); D. B. Lichtenberg, L. J. Tassie, and P. J. Keleman, ibid. 167, 1535 (1968).

[26] M. Shifman and A. Vainshtein, Phys. Rev. D 71, 074010 (2005).

[27] M. Anselmino et al., Rev. Mod. Phys. 65, 1199 (1993).

[28] A. Bender, W. Detmold, A. W. Thomas, and C. D. Roberts, Phys. Rev. C 65, 065203 (2002).

[29] A. Selem and F. Wilczek, arXiv:hep-ph/0602128.

[30] S. Ekelin, S. Fredriksson, M. Jändel, and T. I. Larsson, Phys. Rev. D 28, 257 (1983).

[31] R. L. Jaffe, Phys. Rep. 409, 1 (2005).

[32] C. Alexandrou, Ph. de Forcrand, and B. Lucini, Phys. Rev. Lett. 97, 222002 (2006).

[33] M. Hess, F. Karsch, E. Laermann, and I. Wetzorke, Phys. Rev. D 58, 111502(R) (1998).

[34] D. B. Lichtenberg, W. Namgung, and J. G. Wills, Z. Phys. C 19, 19 (1983).

[35] D. Ebert, T. Feldmann, C. Kettner, and H. Reinhardt, Z. Phys. C 71, 329 (1996).

[36] S. Fleck, B. Silvestre-Brac, and J. M. Richard, Phys. Rev. D 38, 1519 (1988).

[37] E. Hernández, J. Nieves, and J. M. Verde-Velasco, Phys. Lett. B666, 150 (2008).

[38] T. Sjöstrand, S. Mrenna, and P. Skands, J. High Energy Phys. 05 (2006) 026.

[39] C. Amsler et al. (Particle Data Group), Phys. Lett. B667, 1 (2008), http://pdg.lbl.gov.

[40] A. Kushnirenko et al. (SELEX Collaboration), Phys. Rev. Lett. 86, 5243 (2001).
[41] B.-W. Zhang, C. M. Ko, and W. Liu, Phys. Rev. C 77, 024901 (2008).

[42] I. Kuznetsova and J. Rafelski, Eur. Phys. J. C 51, 113 (2007).

[43] A. Andronic, P. Braun-Munzinger, K. Redlich, and J. Stachel, Phys. Lett. B659, 149 (2008).

[44] Y. Oh, B.-Y. Park, and D.-P. Min, Phys. Rev. D 49, 4649 (1994); 50, 3350 (1994); Y. Oh and B.-Y. Park, ibid. 53, 1605 (1996).

[45] T. Aaltonen et al. (CDF Collaboration), Phys. Rev. Lett. 99, 202001 (2007).

[46] M. Karliner, B. Keren-Zur, H. J. Lipkin, and J. L. Rosner, Ann. Phys. (NY) 324, 2 (2009).

[47] L.-W. Chen and C. M. Ko, Phys. Rev. C 73, 044903 (2006).

[48] V. Greco, C. M. Ko, and R. Rapp, Phys. Lett. B595, 202 (2004).

[49] C.-W. Hwang, Eur. Phys. J. C 23, 585 (2002).

[50] B. Silvestre-Brac, Few-Body Syst. 20, 1 (1996).

[51] L. W. Chen, C. M. Ko, W. Liu, and M. Nielsen, Phys. Rev. C 76, 014906 (2007)

[52] A. J. Baltz and C. Dover, Phys. Rev. C 53, 362 (1996).

[53] Y. Oh and C. M. Ko, Phys. Rev. C 76, 054910 (2007).

[54] L. W. Chen, V. Greco, C. M. Ko, S. H. Lee, and W. Liu, Phys. Lett. B601, 34 (2004).

[55] R. T. Cahill, C. D. Roberts, and J. Praschifka, Phys. Rev. D 36, 2804 (1987).

[56] M. Cacciari, P. Nason, and R. Vogt, Phys. Rev. Lett. 95, 122001 (2005).

[57] J. Adams et al. (STAR Collaboration), Phys. Rev. Lett. 94, 062301 (2005).

[58] D. H. Lu, K. Tsushima, A. W. Thomas, A. G. Williams, and K. Saito, Nucl. Phys. A634, 443 (1998); X. Jin and B. K. Jennings, Phys. Rev. C 54, 1427 (1996).

[59] Y. Kanada-En'yo and B. Müller, Phys. Rev. C 74, 061901(R) (2006).

[60] S. S. Adler et al. (PHENIX Collaboration), Phys. Rev. C 71, 051902(R) (2005).

[61] J. Adams et al. (STAR Collaboration), arXiv:nucl-ex/0601042.

[62] P. Sorensen, J. Phys. G 32, S135 (2006).

[63] B. Zhang, C. M. Ko, B.-A. Li, and Z. Lin, Phys. Rev. C 61, 067901 (2000).

[64] Z.-W. Lin, C. M. Ko, B. A. Li, B. Zhang, and S. Pal, Phys. Rev. C 72, 064901 (2005).

[65] W. Liu and C. M. Ko, Phys. Lett. B533, 259 (2002); W. Liu, C. M. Ko, and S. H. Lee, Nucl. Phys. A728, 457 (2003).

[66] Z. Lin, C. M. Ko, and B. Zhang, Phys. Rev. C 61, 024904 (2000); Z. Lin, T. G. Di, and C. M. Ko, Nucl. Phys. A689, 965 (2001).

[67] L. Ravagli and R. Rapp, Phys. Lett. B655, 126 (2007). 